\title{
Large deviation principle for enhanced Gaussian processes
}

\author{
Peter Friz*, Nicolas Victoir \\ Department of Pure Mathematics and Mathematical Statistics, University of Cambridge, Cambridge, UK
}

Received 10 January 2006; received in revised form 2 November 2006; accepted 22 November 2006

Available online 27 February 2007

\begin{abstract}
We study large deviation principles for Gaussian processes lifted to the free nilpotent group of step $N$. We apply this to a large class of Gaussian processes lifted to geometric rough paths. A large deviation principle for enhanced (fractional) Brownian motion, in Hölder- or modulus topology, appears as special case.
\end{abstract}

(c) 2007 Elsevier Masson SAS. All rights reserved.

\section{Résumé}

Nous etudions les principes de grandes deviations pour les «rough paths Gaussiens», pour une large classe de processus Gaussiens. Cette classe contient le mouvement brownien fractionnaire.

() 2007 Elsevier Masson SAS. All rights reserved.

MSC: 60F10; 60G15; 60H99

Keywords: Rough paths; Large deviation principle; Gaussian processes; Fractional Brownian motion

\section{Introduction}

We prove large deviation principles for $d$-dimensional Gaussian processes lifted to the free nilpotent group of step $N$. The example we have in mind is a class of zero mean Gaussian processes subject to certain conditions on the covariance, $[5,17]$.

After recalls on nilpotent groups and Wiener Itô chaos, we give simple conditions under which a large deviation principle holds. To illustrate the method, we quickly check these conditions for enhanced Brownian motion (see $[15,10]$ for earlier approaches). We then apply our methodology to the class of enhanced Gaussian processes considered in [5,17]. Enhanced fractional Brownian motion appears as a special case and we strengthen the results in $[18,4]$.

\footnotetext{
* Corresponding author.

E-mail address: P.K.Friz@statslab.cam.ac.uk (P. Friz).
} 


\subsection{The free nilpotent group and rough paths}

Let $N \geqslant 0$. The truncated tensor algebra of degree $N$ is given by the direct sum

$$
T^{N}\left(\mathbb{R}^{d}\right)=\mathbb{R} \oplus \mathbb{R}^{d} \oplus \cdots \oplus\left(\mathbb{R}^{d}\right)^{\otimes N} .
$$

With the usual scalar product, vector addition, and tensor product $\otimes$ the space $T^{N}\left(\mathbb{R}^{d}\right)$ is an algebra. Let $\pi_{i}$ denote the canonical projection from $T^{N}\left(\mathbb{R}^{d}\right)$ onto $\left(\mathbb{R}^{d}\right)^{\otimes i}$.

Let $q \in[1,2)$ and $x \in C_{0}^{q-v a r}\left([0,1], \mathbb{R}^{d}\right)$, the space of continuous $\mathbb{R}^{d}$-valued paths of bounded $q$-variation started at the origin. The lift $S_{N}(x):[0,1] \rightarrow T^{N}\left(\mathbb{R}^{d}\right)$ is defined as

$$
S_{N}(x)_{t}=1+\sum_{i=1}^{N} \int_{0<s_{1}<\cdots<s_{i}<t} \mathrm{~d} x_{s_{1}} \otimes \cdots \otimes \mathrm{d} x_{s_{i}},
$$

the iterated integrals are Young integrals. Then

$$
G^{N}\left(\mathbb{R}^{d}\right)=\left\{g \in T^{N}\left(\mathbb{R}^{d}\right): \exists x \in C_{0}^{1-\operatorname{var}}\left([0,1], \mathbb{R}^{d}\right): g=S_{N}(x)_{1}\right\}
$$

is a submanifold of $T^{N}\left(\mathbb{R}^{d}\right)$ and, in fact, a Lie group with product $\otimes$, called the free nilpotent group of step $N$. The dilation operator $\delta: \mathbb{R} \times G^{N}\left(\mathbb{R}^{d}\right) \rightarrow G^{N}\left(\mathbb{R}^{d}\right)$ is defined by

$$
\pi_{i}\left(\delta_{\lambda}(g)\right)=\lambda^{i} \pi_{i}(g), \quad i=0, \ldots, N,
$$

and a continuous norm on $G^{N}\left(\mathbb{R}^{d}\right)$, homogeneous with respect to $\delta$, is given

$$
\|g\|=\inf \left\{\operatorname{length}(x): x \in C_{0}^{1-\operatorname{var}}\left([0,1], \mathbb{R}^{d}\right), S_{N}(x)_{1}=g\right\} .
$$

By equivalence of such norms there exists a constant $K_{N}$ such that

$$
\frac{1}{K_{N}} \max _{i=1, \ldots, N}\left|\pi_{i}(g)\right|^{1 / i} \leqslant\|g\| \leqslant K_{N} \max _{i=1, \ldots, N}\left|\pi_{i}(g)\right|^{1 / i} .
$$

The norm $\|\cdot\|$ induces a metric on $G^{N}\left(\mathbb{R}^{d}\right)$ known as Carnot-Caratheodory metric,

$$
d(g, h)=\left\|g^{-1} \otimes h\right\| .
$$

Let $x, y \in C_{0}\left([0,1], G^{N}\left(\mathbb{R}^{d}\right)\right)$, the space of continuous $G^{N}\left(\mathbb{R}^{d}\right)$-valued paths started at the neutral element of $\left(G^{N}\left(\mathbb{R}^{d}\right), \otimes\right)$. We define a supremum distance,

$$
d_{\infty}(x, y)=\sup _{t \in[0,1]} d\left(x_{t}, y_{t}\right)
$$

a Hölder distance, $\alpha \in[0,1]$,

$$
d_{\alpha \text {-Hölder }}(x, y)=\sup _{0 \leqslant s<t \leqslant 1} \frac{d\left(x_{s}^{-1} \otimes x_{t}, y_{s}^{-1} \otimes y_{t}\right)}{|t-s|^{\alpha}},
$$

write

$$
\|x\|_{\alpha \text {-Hölder }}=\sup _{0 \leqslant s<t \leqslant 1} \frac{\left\|x_{s}^{-1} \otimes x_{t}\right\|}{|t-s|^{\alpha}}=\sup _{0 \leqslant s<t \leqslant 1} \frac{d\left(x_{s}, x_{t}\right)}{|t-s|^{\alpha}},
$$

and Lip instead of 1-Hölder. Similarly, one defines $p$-variation regularity and distance for $G^{N}\left(\mathbb{R}^{d}\right)$-valued paths. We refer to [11] for a more detailed discussion of these topics.

The interest for all this comes from T. Lyons' rough path theory, [16,17], a deterministic theory of control differential equations ("Rough Differential Equation"), driven by a continuous $G^{[p]}\left(\mathbb{R}^{d}\right)$-valued path of finite $p$-variation. Brownian motion and Lévy's area can be viewed as a $G^{[p]}\left(\mathbb{R}^{d}\right)$-valued path of finite $p$-variation with $p>2$ and the corresponding RDE solution turns out to be a classical Stratonovich SDE solution. This gives a general recipe how to make sense of differential equations driven by an arbitrary stochastic process $X$ with continuous sample path of finite $p$-variation: find an appropriate lift of $X$ to a $G^{[p]}\left(\mathbb{R}^{d}\right)$-valued path $\mathbf{X}$ with finite $p$-variation use Lyons' machinery. 


\subsection{Gaussian process}

The Wiener space $C_{0}\left([0,1], \mathbb{R}^{d}\right)$ is the space of continuous function started at 0 ; the sup norm induces a topology and hence a Borel $\sigma$-algebra. We assume there is a probability measure $\mathbb{P}$ such that the coordinate process $X$, defined by $X(\omega)_{t}=\omega(t)$, is a centered Gaussian process with covariance $c(s, t)$. We also define $\mathcal{H}$ to be the reproducing kernel Hilbert space associated to $\mathbb{P}$, i.e. the closure of the set of the linear span of the functions $s \rightarrow c\left(t_{i}, s\right)$, under the Hilbert product

$$
\left\langle c\left(t_{i}, \cdot\right), c\left(t_{j}, \cdot\right)\right\rangle=c\left(t_{i}, t_{j}\right) .
$$

Let $i$ denote the canonical injection from the reproducing kernel Hilbert space to the Wiener space. We define $\mathcal{C}_{n}(B)$ the $B$-valued homogeneous Wiener chaos of degree $n$, where $\left(B,|\cdot|_{B}\right)$ is a given Banach space. We also define $C_{n}(B)$ the sum of the first $n$ homogeneous Wiener chaos. We refer to [14,1-3] for more details on Gaussian spaces and Wiener chaos.

\subsection{Enhanced Gaussian process}

We make the assumption that there exists a lift of the Gaussian process $X$ to a process $\mathbf{X}$ with values in $G^{N}\left(\mathbb{R}^{d}\right)$ for some fixed $N \geqslant 1$. Such lifts have been constructed first by Coutin and Qian [5] as a.s. limits in $p$-variation of (canonically lifted) dyadic piecewise linear approximations of $X$. We shall be less specific here and extract those properties of the approximations that we need in the sequel. We make the following

Assumption. There exists a sequence of continuous linear maps $\left\{\Phi_{m}\right\}$ from $C\left([0,1], \mathbb{R}^{d}\right)$ (with uniform topology) onto the space of bounded variation path from $[0,1]$ into $\mathbb{R}^{d}$ (with 1-variation topology) such that

(1) $\lim _{m \rightarrow \infty} \Phi_{m}(x)=x$ for all continuous paths $x$ with respect to uniform topology;

(2) the uniform distance between $S_{N} \circ \Phi_{m}(X)$ and $\mathbf{X}$ converges to 0 in probability.

In fact, Condition (2) is equivalent to the seemingly stronger condition of $L^{p}$-convergence.

Lemma 1. Convergence of $d_{\infty}\left(S_{N} \circ \Phi_{m}(X), \mathbf{X}\right)$ to zero in probability is equivalent to convergence in $L^{p}$ for all $p \in[1, \infty)$.

Proof. Theorem III.2 in [20] and Eq. (1.1).

Remark 1. Assumption 2 is always satisfied when $N=1$. In the applications discussed in later sections, $N$ will be related to the regularity of $X$.

Example 1. Let $\left\{\Phi_{m}\right\}$ be the piecewise linear approximations based on a sequence of dissections $\left\{D_{m}\right\}$ with mesh $\left|D_{m}\right| \rightarrow 0$. If $X$ denotes fractional Brownian motion of parameter $H>\frac{1}{4}$, then the lift $\mathbf{X}$ for $N=[1 / H]$ and was first constructed in [5], see also [4].

Remark 2. As is well known (e.g. Theorem 1 in [13]) any continuous Gaussian process $X$ on $[0,1]$ is the uniform limit of

$$
X_{t}^{(m)} \equiv \sum_{j=1}^{m} \xi_{j}(\omega) \psi_{j}(t)
$$

where $\left\{\psi_{j}\right\}$ is an orthonormal basis for the reproducing Kernel Hilbert space $\mathcal{H}$ and the $\left\{\xi_{j}\right\}$ are i.i.d. standard Gaussian given by the image of $\psi_{j}$ under the isometric isomorphism $\theta: \mathcal{H} \rightarrow \mathcal{L}_{2}\left(X_{t}: t \in[0,1]\right)$, the closure of $\left\{X_{t}:[0,1]\right\}$ in $L^{2}(\mathbb{P})$. The Hilbert structure of $\mathcal{H}$ implies that for all $h \in \mathcal{H}$,

$$
h^{(m)} \equiv \sum_{j=1}^{m}\left\langle\psi_{j}, h\right\rangle \psi_{j} \rightarrow h \quad \text { in } \mathcal{H}
$$


and hence uniformly. The construction of $\mathbf{X}$ can be based on such (or similar) approximations, ${ }^{1}$ see $[6,11,19,8]$, but they do not satisfy our assumption. It may be possible to adapt the subsequent proofs, aimed to establish a large deviation principle for $\mathbf{X}$, to such approximations. As this requires further work without improving the results we shall not pursue this further here.

\section{Wiener chaos and $G^{N}\left(\mathbb{R}^{d}\right)$-valued paths}

The hypercontractivity of the Ornstein-Uhlenbeck semigroup leads to a useful

Lemma 2. Let $Z_{n}$ be a random variable in $\mathcal{C}_{n}(B)$. Assume $1<p<q<\infty$. Then

$$
\left\|Z_{n}\right\|_{L^{q}(\mathbb{P} ; B)} \leqslant\left(\frac{q-1}{p-1}\right)^{n / 2}\left\|Z_{n}\right\|_{L^{p}(\mathbb{P} ; B)} .
$$

Proof. Let $P_{t}$ denote the Ornstein-Uhlenbeck semigroup. Whenever $1<p<q<\infty$ and $t>0$ satisfies

$$
\mathrm{e}^{t} \geqslant\left(\frac{q-1}{p-1}\right)^{1 / 2}
$$

then, for all $f \in L^{p}(\mathbb{P} ; B)$,

$$
\left\|P_{t} f\right\|_{L^{q}} \leqslant\|f\|_{L^{p}}
$$

We also recall that

$$
P_{t} Z_{n}=\mathrm{e}^{-n t} Z_{n}
$$

See [14] for this two results. We now choose $t$ such that equality holds in (2.1) and find

$$
\left(\frac{q-1}{p-1}\right)^{-n / 2}\left\|Z_{n}\right\|_{L^{q}}=\mathrm{e}^{-n t}\left\|Z_{n}\right\|_{L^{q}}=\left\|P_{t} Z_{n}\right\|_{L^{q}} \leqslant\left\|Z_{n}\right\|_{L^{p}} .
$$

It is well known that $L^{p}(\mathbb{P} ; B)$ - and $L^{q}(\mathbb{P} ; B)$-norms are equivalent on

$$
C_{n}(B)=\mathcal{C}_{1}(B)+\cdots+\mathcal{C}_{n}(B) .
$$

The next lemma quantifies this equivalence.

Lemma 3. Let $n \in \mathbb{N}$ and $Z$ be a random variable in $C_{n}(B)$. Assume $2 \leqslant p \leqslant q<\infty$. Then there exists a constant $M_{n}$ such that

$$
\|Z\|_{L^{p}(\mathbb{P} ; B)} \leqslant\|Z\|_{L^{q}(\mathbb{P} ; B)} \leqslant M_{n}(q-1)^{n / 2}\|Z\|_{L^{p}(\mathbb{P} ; B)} .
$$

Proof. Only the second inequality requires a proof. Write $Z=\sum_{i=0}^{n} Z_{i}$ with $Z_{i} \in \mathcal{C}_{i}$. From Lemma 2, for all $i \leqslant n$,

$$
\left\|Z_{i}\right\|_{L^{q}(\mathbb{P} ; B)} \leqslant\left(\frac{q-1}{p-1}\right)^{i / 2}\left\|Z_{i}\right\|_{L^{p}(\mathbb{P} ; B)} .
$$

The sum $C_{n}(B)=\mathcal{C}_{1}(B)+\cdots+\mathcal{C}_{n}(B)$ is topological direct in $L^{0}(\mathbb{P} ; B)$, see [3, p. 6] for instance, which implies that the projection $Z \mapsto Z_{i}$ is continuous in $L^{2}(\mathbb{P} ; B)$. It follows that $\left\|Z_{i}\right\|_{L^{2}(\mathbb{P} ; B)} \leqslant c\|Z\|_{L^{2}(\mathbb{P} ; B)}$ for some constant $c=c(n)$. Then, for $p \geqslant 2$,

$$
\left\|Z_{i}\right\|_{L^{p}(\mathbb{P} ; B)} \leqslant(p-1)^{i / 2}\left\|Z_{i}\right\|_{L^{2}(\mathbb{P} ; B)} \leqslant c(p-1)^{i / 2}\|Z\|_{L^{2}(\mathbb{P} ; B)} \leqslant c(p-1)^{i / 2}\|Z\|_{L^{p}(\mathbb{P} ; B)} .
$$

By Hölder's inequality for finite sums,

$$
|Z|_{B}^{q} \leqslant n^{q-1} \sum_{i=0}^{n}\left|Z_{i}\right|_{B}^{q}
$$

\footnotetext{
1 At least for, $N \leqslant 2$ it is shown in [6] that reproducing kernel - and piecewise linear approximations yield the same lifted process.
} 
and after taking expectations,

$$
\|Z\|_{L^{q}(\mathbb{P} ; B)} \leqslant n^{1-1 / q}\left(\sum_{i=0}^{n}\left\|Z_{i}\right\|_{L^{q}(\mathbb{P} ; B)}^{q}\right)^{1 / q} .
$$

Hence, from (2.2) and (2.3),

$$
\begin{aligned}
\|Z\|_{L^{q}(\mathbb{P} ; B)} & \leqslant c n^{1-1 / q}\left(\sum_{i=0}^{n}\left(\frac{q-1}{p-1}\right)^{q i / 2}\left\|Z_{i}\right\|_{L^{p}(\mathbb{P} ; B)}^{q}\right)^{1 / q} \\
& \leqslant c n^{1-1 / q}\left(\sum_{i=0}^{n}(q-1)^{q n / 2}\|Z\|_{L^{p}(\mathbb{P} ; B)}^{q}\right)^{1 / q} \\
& \leqslant c n(q-1)^{n / 2}\|Z\|_{L^{p}(\mathbb{P} ; B) .}
\end{aligned}
$$

Corollary 1. Let $X, Y$ be two continuous $G^{N}\left(\mathbb{R}^{d}\right)$-valued processes such that, for each $i=1, \ldots, N$, the projection

$$
t \mapsto \pi_{i}\left(X_{t}\right), \quad \pi_{i}\left(Y_{t}\right) \in\left(\mathbb{R}^{d}\right)^{\otimes i}
$$

belongs to $C_{i}\left(B_{i}\right)=\mathcal{C}_{0}\left(B_{i}\right) \oplus \mathcal{C}_{1}\left(B_{i}\right) \oplus \cdots \oplus \mathcal{C}_{n}\left(B_{i}\right)$, where

$$
B_{i}=C_{0}\left([0,1],\left(\mathbb{R}^{d}\right)^{\otimes i}\right)
$$

and $\mathcal{C}_{j}\left(B_{i}\right)$ is the $B_{i}$-valued homogeneous Wiener chaos of degree $j$. Assume $2<q<\infty$. Then there exists a constant $M_{N}$ such that

$$
\left\|d_{\infty}(X, Y)\right\|_{L^{2 N}(\mathbb{P})} \leqslant\left\|d_{\infty}(X, Y)\right\|_{L^{q N}(\mathbb{P})} \leqslant \sqrt{q} M_{N}\left\|d_{\infty}(X, Y)\right\|_{L^{2 N}(\mathbb{P})} .
$$

Proof. Again, only the second inequality requires a proof. For $i=1, \ldots, N$ define

$$
Z_{i}: t \mapsto \pi_{i}\left(X_{t}^{-1} \otimes Y_{t}\right) \in\left(\mathbb{R}^{d}\right)^{\otimes i} .
$$

Observe that $Z_{i} \in C_{i}\left(B_{i}\right) \subset C_{N}\left(B_{i}\right)$. Let $|\cdot|_{\infty}$ denote the supremum norm on $\left(\mathbb{R}^{d}\right)^{\otimes i}$-valued paths. From Eq. (1.1),

$$
\frac{1}{K_{N}} \max _{i=1, \ldots, N}\left|Z_{i}\right|_{\infty}^{1 / i} \leqslant d_{\infty}(X, Y) \leqslant K_{N} \max _{i=1, \ldots, N}\left|Z_{i}\right|_{\infty}^{1 / i}
$$

Therefore, for all $q \geqslant 2$,

$$
\frac{1}{K_{N}} \max _{i=1, \ldots, N}\left\|Z_{i}\right\|_{L^{q N / i}(\mathbb{P})}^{1 / i} \leqslant\left\|d_{\infty}(X, Y)\right\|_{L^{q N}(\mathbb{P})} \leqslant K_{N} \max _{i=1, \ldots, N}\left\|Z_{i}\right\|_{L^{q N / i}(\mathbb{P})}^{1 / i} .
$$

From Lemma 3

$$
\begin{aligned}
\left\|Z_{i}\right\|_{L^{q N / i}(\mathbb{P})}^{1 / i} & \leqslant M_{N}^{1 / i} \sqrt{q N / i-1}\left\|Z_{i}\right\|_{L^{2 N / i}(\mathbb{P})}^{1 / i} \\
& \leqslant \sqrt{q} M_{N}^{\prime}\left\|Z_{i}\right\|_{L^{2 N / i}(\mathbb{P})}^{1 / i} \\
& \leqslant \sqrt{q} M_{N}^{\prime \prime}\left\|d_{\infty}(X, Y)\right\|_{L^{2 N}(\mathbb{P})}
\end{aligned}
$$

where we used (2.5) with $q=2$ in the last line. Another look at (2.5) finishes the proof.

\section{Large deviation results for $\left(\delta_{\varepsilon} X\right)_{\varepsilon>0}$}

From general principles, $(\varepsilon X)_{\varepsilon>0}$ satisfies a large deviation principle with good rate function $I$ in uniform topology, where $I$ is given by (see [14] for example):

$$
I(y)= \begin{cases}\frac{1}{2}\|x\|_{\mathcal{H}}^{2} & \text { when } y=i(x) \text { for some } x \in \mathcal{H} \\ +\infty & \text { otherwise. }\end{cases}
$$


It is clear that

$$
S_{N} \circ \Phi_{m}:\left(C\left([0,1], \mathbb{R}^{d}\right),|\cdot|_{\infty}\right) \rightarrow C\left([0,1], G^{N}\left(\mathbb{R}^{d}\right), d_{\infty}\right)
$$

is continuous. By the contraction principle [7], $S_{N}\left(\varepsilon \Phi_{m}(X)\right)$ satisfies a large deviation principle with good rate function

$$
J_{m}(y)=\inf \left\{I(x), x \text { such that } S_{N}\left(\Phi_{m}(x)\right)=y\right\},
$$

the infimum of the empty set being $+\infty$. Essentially, a large deviation principle for $\delta_{\varepsilon} \mathbf{X}$ is obtained by sending $m$ to infinity. To this end we need

Lemma 4. Let $\delta>0$ fixed. Then

$$
\lim _{m \rightarrow \infty} \varlimsup_{\varepsilon \rightarrow 0} \varepsilon^{2} \log \mathbb{P}\left(d_{\infty}\left(S_{N}\left(\Phi_{m}(\varepsilon X)\right), \delta_{\varepsilon} \mathbf{X}\right)>\delta\right)=-\infty
$$

Proof. First observe that

$$
d_{\infty}\left(S_{N}\left(\Phi_{m}(\varepsilon X)\right), \delta_{\varepsilon} \mathbf{X}\right)=\varepsilon d_{\infty}\left(S_{N}\left(\Phi_{m}(X)\right), \mathbf{X}\right) .
$$

By standing assumption on $\mathbf{X}$ and Lemma 1 we have

$$
\alpha_{m}:=\left\|d_{\infty}\left(S_{N}\left(\Phi_{m}(X)\right), \mathbf{X}\right)\right\|_{L^{2 N}} \underset{m \rightarrow \infty}{\longrightarrow} 0 .
$$

Then, by Corollary 1 ,

$$
\left\|d_{\infty}\left(S_{N}\left(\Phi_{m}(X)\right), \mathbf{X}\right)\right\|_{L^{q}} \leqslant M_{N} \sqrt{q} \alpha_{m} \quad \forall q \geqslant 2 N .
$$

We then estimate

$$
\begin{aligned}
\mathbb{P}\left(d_{\infty}\left(S_{N}\left(\Phi_{m}(\varepsilon X)\right), \delta_{\varepsilon} \mathbf{X}\right)>\delta\right) & =\mathbb{P}\left(d_{\infty}\left(S_{N}\left(\Phi_{m}(X)\right), \mathbf{X}\right)>\frac{\delta}{\varepsilon}\right) \\
& \leqslant\left(\frac{\delta}{\varepsilon}\right)^{-q} \sqrt{q}^{q} \alpha_{m}^{q} \\
& \leqslant \exp \left[q \log \left(\frac{\varepsilon}{\delta} \alpha_{m} \sqrt{q}\right)\right],
\end{aligned}
$$

and after choosing $q=1 / \varepsilon^{2}$ we obtain, for $\varepsilon$ small enough,

$$
\varepsilon^{2} \log \mathbb{P}\left(\sum_{t \in D} d\left(S_{N}\left(\Phi_{m}(\varepsilon X)\right)_{t}, \delta_{\varepsilon} \mathbf{X}_{t}\right)>\delta\right) \leqslant \log \left(\frac{\alpha_{m}}{\delta}\right) .
$$

Now take the limits $\overline{\lim }_{\varepsilon \rightarrow 0}$ and $\lim _{m \rightarrow \infty}$ to finish the proof.

The following theorem is a straight forward application of the extended contraction principle [7, Theorem 4.2.23] and Lemma 4 . The point is that although $S_{N}$ is a only a measurable map, defined as a.s. limit, it has exponentially good approximations given by $\left\{S_{N} \circ \Phi_{m}: m \geqslant 1\right\}$.

Theorem 1. Assume that $S_{N}(h)$, defined as the pointwise limit of $\left(S_{N} \circ \Phi_{m}\right)(h)$. when $m \rightarrow \infty$ exists for $h$ such that $I(h)<\infty$ (i.e. all $h$ in the Cameron-Martin space), and that for all $\Lambda>0$,

$$
\lim _{m \rightarrow \infty} \sup _{\{h: I(h) \leqslant \Lambda\}} d_{\infty}\left[\left(S_{N} \circ \Phi_{m}\right)(h), S_{N}(h)\right]=0 .
$$

Then the family $\left(\delta_{\varepsilon} \mathbf{X}\right)_{\varepsilon>0}$ satisfies a large deviation principle in uniform topology with good rate function

$$
J(\mathbf{y})=\inf \left\{I(x), x \text { such that } S_{N}(x)=\mathbf{y}\right\} .
$$

The following corollary allows us to strengthen the topology. 
Corollary 2. Under the above assumptions and the additional condition

$$
\exists \alpha^{\prime}>0, c>0: \mathbb{E}\left(\exp c\|\mathbf{X}\|_{\alpha^{\prime} \text {-Hölder }}^{2}\right)<\infty,
$$

the family $\left(\delta_{\varepsilon} \mathbf{X}\right)_{\varepsilon>0}$ satisfies a large deviation principle in $\alpha$-Hölder topology for all $\alpha \in\left[0, \alpha^{\prime}\right)$ with good rate function $J(\mathbf{y})$.

Proof. By the inverse contraction principle [7, Theorem 4.2.4.] it suffices to show that the laws of $\left(\delta_{\varepsilon} \mathbf{X}\right)$ are exponentially tight in $\alpha$-Hölder topology. But this follows from the compact embedding of

$$
C^{\alpha^{\prime} \text {-Hölder }}\left([0,1], G^{N}\left(\mathbb{R}^{d}\right)\right) \subset \subset C^{\alpha-H o ̈ l d e r}\left([0,1], G^{N}\left(\mathbb{R}^{d}\right)\right)
$$

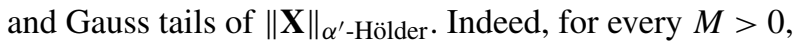

$$
K_{M}=\left\{x:\|x\|_{\alpha^{\prime}-\text { Hölder }} \leqslant M\right\}
$$

defines a precompact set w.r.t. $\alpha$-Hölder topology. Then

$$
\begin{aligned}
\varepsilon^{2} \log \left[\left(\delta_{\varepsilon} \mathbf{X}\right)_{*} \mathbb{P}\right]\left(K_{M}^{c}\right) & =\varepsilon^{2} \log \mathbb{P}\left[\left\|\delta_{\varepsilon} \mathbf{X}\right\|_{\alpha^{\prime} \text {-Hölder }}>M\right] \\
& =\varepsilon^{2} \log \mathbb{P}\left[\|\mathbf{X}\|_{\alpha^{\prime} \text {-Hölder }}>M / \varepsilon\right] \\
& \lesssim-M^{2} . \quad \square
\end{aligned}
$$

Remark 3. The same proof applies to the (weaker) $p$-variation topology and the (stronger) modulus topology considered in [10].

We emphasise the generality of this approach: a large deviation result for an enhanced Gaussian process holds provided (a) the enhanced Gaussian process exists and (b) a condition on the reproducing Kernel of the Gaussian process.

As a warm-up we show how to apply this to enhanced Brownian motion. We will then discuss a wider class of Gaussian process, which contains fractional Brownian motion with Hurst parameter $H>\frac{1}{4}$.

\section{LDP for enhanced Brownian motion}

Let $X=B$ is be $d$-dimensional Brownian motion. We take $\Phi_{m}$ be the piecewise linear approximation over at the dyadic dissection of $[0,1]$ with mesh $2^{-m}$. Then $S_{2}\left(\Phi_{m}(B)\right)$ converges uniformly on $[0,1]$ and in probability to a process $\mathbf{B}$, see [17,9,10]). Large deviations for $\left(\delta_{\varepsilon} \mathbf{B}\right)_{\varepsilon}$ were first obtained in $p$-variation topology [15], then in Hölder and modulus topology [10]. The following proof is considerably quicker. There are only two things to check.


scaling $d\left(\mathbf{B}_{s}, \mathbf{B}_{t}\right) \stackrel{\mathcal{D}}{=}|t-s|^{1 / 2}\left\|\mathbf{B}_{0,1}\right\|$, noting that $\left\|\mathbf{B}_{0,1}\right\|$ has Gauss tails, followed by an application of the GarsiaRodemich-Rumsey lemma. One could prove the result directly by extending Lemma 1 to $\alpha$-Hölder metric.

(2) Condition (3.2). But this is a simple consequence of the fact that the 1-variation norm of $h$ is bounded by $\sqrt{2 I(h)}$ and that the 1-variation of $\Phi_{m}(h)$ is bounded by the 1-variation of $h$. From Theorem 1 and Corollary 2 we deduce

Theorem 2. Let $\mathbf{B}=\mathbf{B}(\omega) \in C\left([0,1], G^{2}\left(\mathbb{R}^{d}\right)\right)$ denote enhanced Brownian Motion. Let $\alpha \in[0,1 / 2)$. Then $\left(\delta_{\varepsilon} \mathbf{B}\right)_{\varepsilon>0}$ satisfies a large deviation principle in $\alpha$-Hölder topology with good rate function

$$
J(\mathbf{y})=\inf \left\{I(x), x \text { such that } S_{N}(x)=\mathbf{y}\right\} .
$$

When restricting $\alpha$ to $\alpha \in(1 / 3,1 / 2)$ the universal limit theorem gives the Freidlin-Wentzell theorem (in $\alpha$-Hölder topology) as corollary. See $[15,10,4]$ for details on this by now classical application of Lyons' limit theorem.

Remark 4. The case of enhanced fractional Brownian motion, $H>\frac{1}{4}$, is similar. First, Gauss tails of the $\alpha$-Hölder norm, for any $\alpha \in[0, H)$, follow from scaling just as above. (This has already been used in [11,4].) Secondly, condition (3.2) follows from the fact that the Cameron-Martin space for fBM is embedded in the space of finite $q$-variation for 
any $q$ less than $1 /(1 / 2+H)$, see [12]. In any case, enhanced fractional Brownian motion is a special case of the processes considered in the next section.

\section{LDP for a class of Gaussian processes}

We fix a parameter $H>0$. We now specialise to zero-mean $\mathbb{R}^{d}$-valued Gaussian processes $\left\{X_{t}: t \in[0,1]\right\}$ with independent components $x=X^{i}, i=1, \ldots, d$, satisfying

$$
\begin{aligned}
& E\left(\left|x_{s, t}\right|^{2}\right) \leqslant c|t-s|^{2 H}, \quad \text { for all } s<t, \\
& \left|E\left(x_{s, s+h} x_{t, t+h}\right)\right| \leqslant c|t-s|^{2 H}\left|\frac{h}{t-s}\right|^{2}, \quad \text { for all } s, t, h \text { with } h<t-s
\end{aligned}
$$

for some constant $c>0$. Note that such a process has a.s. $1 / p$-Hölder continuous sample paths when $p>1 / H$. A particular example of such a Gaussian process is fractional Brownian motion of Hurst parameter $H$. This condition is the one which allowed Coutin and Qian [5] to construct the lift of $X$ to a rough path. As before $\mathcal{H}$ denotes the Cameron-Martin space. We take $\Phi_{m}$ to be the piecewise linear approximation over at the dyadic dissections of $[0,1]$ with mesh $2^{-m}$.

Definition 1. For $H>1 / 4$, we define, according to [5], the enhanced Gaussian process $\mathbf{X}$ to be the (a.s. and in $L^{q}$ for all $q$ ) limit in $1 / p$-Hölder norm of $S_{[p]}\left(\Phi_{m}(X)\right)$, where $1 / p<H .^{2}$

To obtain the large deviation principle for $\left(\delta_{\varepsilon} \mathbf{X}\right)_{\varepsilon>0}$ there are, as in the case of EBM, only two things to check.

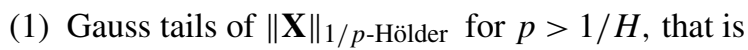

$$
\mathbb{E} \exp \left(\alpha\|\mathbf{X}\|_{1 / p \text {-Hölder }}^{2}\right)<\infty .
$$

First note that for all $q \geqslant 1$,

$$
\sup _{0 \leqslant s<t \leqslant 1} \mathbb{E}\left[\left(\frac{\left\|\mathbf{X}_{s, t}\right\|}{|t-s|^{H}}\right)^{q}\right]<\infty .
$$

Similar to Corollary 1 , for all $s, t$, and $k \geqslant 1$,

$$
\mathbb{E}\left(\left|\frac{\left\|\mathbf{X}_{s, t}\right\|}{|t-s|^{H}}\right|^{2 k}\right)^{1 / 2 k} \leqslant C \sqrt{k} E\left(\left|\frac{\left\|\mathbf{X}_{s, t}\right\|}{|t-s|^{H}}\right|^{q}\right)^{1 / q},
$$

where $q$ is any fixed real greater than $2[1 / H]$. Now expand the exponential in a power series to see that there exists $\alpha>0$ s.t. $\mathbb{E} \exp \left(\alpha\left\|\mathbf{X}_{s, t}\right\|^{2} /|t-s|^{2 H}\right)<\infty$ for all $s, t$. The proof of 5.3 is then finished using the GarsiaRodemich-Rumsey lemma, just as in [10].

(2) Condition (3.2). We show (a) that for some $q \in[1,2)$ the $q$-variation norm of a Cameron-Martin path is bounded by a constant times its Cameron-Martin norm and (b) a uniform modulus of continuity for elements in $\{h: I(h) \leqslant \Lambda\}$. A soft argument, spelled out in Theorem 3 below, will then imply (3.2).

We start by proving that the quadratic variation of Cameron-Martin paths converges exponentially fast to 0 .

Lemma 5. Let $H \in(1 / 4,1)$. Then there exists a constant $C_{H, c}$ such that for all $n \geqslant 0$ and all $h \in \mathcal{H}$

$$
\sum_{k=0}^{2^{n}-1}\left|h_{\frac{k}{2^{n}}, \frac{k+1}{2^{n}}}\right|^{2} \leqslant C_{H, c}|h|_{\mathcal{H}}^{2} 2^{n(1-4 H) / 2} .
$$

$\overline{2}$ The convergence in was proven in $p$-variation topology, but it is obvious when reading the proof that it also holds in Hölder norm. 
Proof. Without loss of generalities, we assume $d=1$. Write $R(s, t)=\mathbb{E}\left(X_{s} X_{t}\right)$ for the covariance. The CameronMartin space is the closure of functions of form $h=\sum_{i} a_{i} R\left(t_{i}, \cdot\right)$ with respect to the norm

$$
|h|_{\mathcal{H}}=\left|\sum_{i} a_{i} R\left(t_{i}, \cdot\right)\right|_{\mathcal{H}}^{2}=\sum_{i, j} a_{i} a_{j} R\left(t_{i}, t_{j}\right)=\mathbb{E}\left(Z^{2}\right)
$$

where $Z$ is the zero-mean Gaussian r.v. given by $Z=\sum_{i} a_{i} X_{t_{i}}$. Observe that $h_{s, t}=\mathbb{E}\left(Z X_{s, t}\right)$. We keep $n$ fixed for the rest of the proof.

$$
Q_{n}:=\sum_{k=0}^{2^{n}-1}\left|h_{\frac{k}{2^{n}}, \frac{k+1}{2^{n}}}\right|^{2}=\sum_{k=0}^{2^{n}-1} \mathbb{E}\left(X_{\frac{k}{2^{n}}, \frac{k+1}{2^{n}}} Z\right)^{2} .
$$

Note that if $U, V$ are two zero-mean Gaussian random variables, then

$$
\mathbb{E}\left(U^{2} V^{2}\right)=2 \mathbb{E}(U V)^{2}+\mathbb{E}\left(U^{2}\right) \mathbb{E}\left(V^{2}\right) \quad \text { or } \quad \operatorname{Cov}\left(U^{2}, V^{2}\right)=2 \mathbb{E}(U V)^{2} .
$$

This allows us to write

$$
\begin{aligned}
Q_{n} & =\frac{1}{2} \sum_{k=0}^{2^{n}-1} \mathbb{E}\left(Z^{2}\left[X_{\frac{k}{2^{n}}, \frac{k+1}{2^{n}}}^{2}-\mathbb{E}\left(X_{\frac{k}{2^{n}}, \frac{k+1}{2^{n}}}^{2}\right)\right]\right) \\
& =\frac{1}{2} \mathbb{E}\left(Z^{2} \sum_{k=0}^{2^{n}-1}\left[X_{\frac{k}{2^{n}}, \frac{k+1}{2^{n}}}^{2}-\mathbb{E}\left(X_{\frac{k}{2^{n}}, \frac{k+1}{2^{n}}}^{2}\right)\right]\right) \\
& \leqslant \frac{1}{2} \mathbb{E}\left(Z^{4}\right)^{1 / 2}\left(\sum_{k, l=0}^{2^{n}-1} \operatorname{Cov}\left(X_{\frac{k}{2^{n}}, \frac{k+1}{2^{n}}}^{2}, X_{\frac{l}{2^{n}}, \frac{l+1}{2^{n}}}^{2}\right)\right)^{1 / 2} \\
& =\sqrt{\frac{3}{2}} \mathbb{E}\left(Z^{2}\right)\left(\sum_{k, l=0}^{2^{n}-1} \mathbb{E}\left(X_{\frac{k}{2^{n}},}, \frac{k+1}{2^{n}} X_{\frac{l}{2^{n}},}, \frac{l+1}{2^{n}}\right)^{2}\right)^{1 / 2}
\end{aligned}
$$

where we used (5.4) again. The double sum in line just above splits naturally in a diagonal part, where $k=l$, and twice the sum over $k<l$. With (5.1) we control the diagonal part,

$$
\sum_{k=0}^{2^{n}-1} \mathbb{E}\left(X_{\frac{k}{2^{n}}, \frac{k+1}{2^{n}}}^{2}\right)^{2} \leqslant c^{2} 2^{n(1-4 H)} .
$$

The other part is estimated by using (5.2). Indeed,

$$
\begin{aligned}
\sum_{0 \leqslant k<l \leqslant 2^{n}-1} \mathbb{E}\left(X_{\frac{k}{2^{n}}, \frac{k+1}{2^{n}}} X_{\frac{l}{2^{n}}, \frac{l+1}{2^{n}}}\right)^{2} & =\sum_{k=0}^{2^{n}-1} \sum_{m=1}^{2^{n}-k-1} \mathbb{E}\left(X_{\frac{k}{2^{n}}, \frac{k+1}{2^{n}}} X_{\frac{k+m}{2^{n}}, \frac{k+m+1}{2^{n}}}\right)^{2} \\
& \leqslant c^{2} \sum_{k=0}^{2^{n}-1} \sum_{m=1}^{2^{n}-k-1}\left|\frac{m}{2^{n}}\right|^{4 H-4} 2^{-4 n} \\
& \leqslant\left(\sum_{m=1}^{\infty} m^{4 H-4}\right) c^{2} 2^{n(1-4 H)} .
\end{aligned}
$$

Putting everything together yields a constant $C=C(H, c)$ such that

$$
Q_{n} \leqslant C_{H} \mathbb{E}\left(Z^{2}\right) 2^{n(1-4 H) / 2}=C_{H, c}|h|_{\mathcal{H}} 2^{n(1-4 H) / 2} .
$$

This finishes the proof.

Corollary 3. Let $H \in(1 / 4,1 / 2]$ and $q \in(1 \vee 1 /(H+1 / 4), 2)$. Then $\mathcal{H}$ is continuously embedded in $C_{0}^{q-v a r}\left([0,1], \mathbb{R}^{d}\right)$. More precisely, there exists a constant $C_{H, q, c}$ such that for all $h \in \mathcal{H}$,

$$
|h|_{q-\mathrm{var}} \leqslant C_{H, q, c}|h|_{\mathcal{H}} .
$$


Proof. We use the inequality

$$
|h|_{q \text {-var }}^{q} \leqslant C_{\gamma, q} \sum_{n=1}^{\infty} n^{\gamma} \sum_{k=0}^{2^{n}-1}\left|h_{\frac{k}{2^{n}}, \frac{k+1}{2^{n}}}\right|^{q},
$$

see [17] for example. From the previous lemma and Hölder's inequality,

$$
\begin{aligned}
\sum_{k=0}^{2^{n}-1}\left|h_{\frac{k}{2^{n}}, \frac{k+1}{2^{n}}}\right|^{q} & \leqslant\left(\sum_{k=0}^{2^{n}-1}\left|h_{\frac{k}{2^{n}}, \frac{k+1}{2^{n}}}\right|^{2}\right)^{q / 2} 2^{n(1-q / 2)} \\
& \leqslant C|h|_{\mathcal{H}}^{q} 2^{n\left[(1-4 H) \frac{q}{4}+1-\frac{q}{2}\right]} .
\end{aligned}
$$

Observe

$$
(1-4 H) \frac{q}{4}+1-\frac{q}{2}<0 \Leftrightarrow q\left(H+\frac{1}{4}\right)>1 .
$$

Hence, for every $q \geqslant 1$ such that $q>1 /(H+1 / 4)$, we have

$$
|h|_{q-\mathrm{var}} \leqslant C_{H, q, c}|h|_{\mathcal{H}} .
$$

This result is not optimal. Cameron-Martin paths associated to usual Brownian motion are of bounded variation (三 finite 1-variation) whereas the above corollary applied to $H=1 / 2$ only gives finite $q$-variation for $q>4 / 3$. In [12], we prove that Cameron-Martin paths associated to fractional Brownian motion are of finite $q$-variation for any $q$ greater than $1 \vee 1 /(H+1 / 2)$.

Lemma 6. Let $H \in(0,1)$. Then $\mathcal{H}$ is continuously embedded in $C_{0}^{H-H o ̈ l d e r}([0,1])$. More precisely, there exists a constant $C_{H, c}$ such that for all $h \in \mathcal{H}$,

$$
|h|_{H \text {-Hölder }} \leqslant C_{H, c}|h|_{\mathcal{H}} \text {. }
$$

Proof. We use the notation of the above proofs. In particular, $h_{s, t}=\mathbb{E}\left(Z X_{s, t}\right)$. By Cauchy-Schwartz and (5.1),

$$
\left|h_{s, t}\right| \leqslant \sqrt{\mathbb{E}\left(Z^{2}\right)} \sqrt{\mathbb{E}\left(X_{s, t}^{2}\right)} \leqslant C|h|_{\mathcal{H}}|t-s|^{H} .
$$

Theorem 3. For any fixed $N \geqslant 1$, for all $\Lambda>0$,

$$
\lim _{m \rightarrow \infty} \sup _{\{h: I(h) \leqslant \Lambda\}} d_{\infty}\left[\left(S_{N} \circ \Phi_{m}\right)(h), S_{N}(h)\right]=0 .
$$

Proof. Observe first that for $h$ such that $I(h) \leqslant \Lambda$, we have

$$
\begin{aligned}
\left|\Phi_{m}(h)-h\right|_{\infty} & \leqslant \sup _{|t-s| \leqslant 2^{-m}}\left|h_{s, t}\right| \\
& \leqslant \Lambda^{1 / 2} C_{H} 2^{-m H} .
\end{aligned}
$$

Observe also that for $q \geqslant 1 /(1 / 4+H)$,

$$
\left|\Phi_{m}(h)\right|_{q-\mathrm{var}} \leqslant 3|h|_{q-\mathrm{var}} \leqslant 3 C_{q, h} \Lambda^{1 / 2} .
$$

By interpolation, for $q^{\prime}>q$, we therefore obtain that

$$
\lim _{m \rightarrow \infty} \sup _{\{h: I(h) \leqslant \Lambda\}}\left|\Phi_{m}(h)-h\right|_{q^{\prime}-\mathrm{var}}=0 .
$$

Then using Theorem 2.2.2 in [16] (or just the continuity of the Young integral), we obtain our theorem.

We have therefore the following: 
Corollary 4. Assume that for some $H \in(1 / 4,1)$, the Gaussian process satisfies condition (5.1) and (5.2) and let $\mathbf{X}$ be as in Definition 1. Then, $\left(\delta_{\varepsilon} \mathbf{X}\right)$ satisfies a large deviation principle in $1 / p$-Hölder topology where $p>1 / H$ with good rate function

$$
J(\mathbf{y})=\inf \left\{\frac{1}{2}|x|_{\mathcal{H}}, x \text { such that } S_{[p]}(x)=\mathbf{y}\right\} .
$$

Once again, applying the Itô map to $\delta_{\varepsilon} \mathbf{X}$, we can obtain a generalisation of the Freidlin-Wentzell theorem, i.e. a large deviation principle for solution of stochastic differential equation driven by the enhanced Gaussian process $\mathbf{X}$.

\section{Acknowledgements}

The authors would like to thank Christer Borell, Ben Garling for helpful discussions and an anonymous referee for his/her careful reading. The first author acknowledges partial support from a Leverhulme Research Fellowship.

\section{References}

[1] C. Borell, Tail probabilities in Gauss space, in: Vector Space Measures and Applications, Dublin, 1977, in: Lecture Notes in Math., vol. 644, Springer-Verlag, 1978, pp. 73-82.

[2] C. Borell, On polynomials chaos and integrability, Probab. Math. Statist. 3 (1984) 191-203.

[3] C. Borell, On the Taylor series of a Wiener polynomial, in: Seminar Notes on Multiple Stochastic Integration, Polynomial Chaos and their Integration, Case Western Reserve University, Cleveland, 1984.

[4] L. Coutin, P. Friz, N. Victoir, Good rough path sequences and applications to anticipating \& fractional stochastic calculus, ArXiv-preprint, 2005.

[5] L. Coutin, Z. Qian, Stochastic analysis, rough path analysis and fractional Brownian motions, Probab. Theory Relat. Fields 122 (2002) 108140.

[6] L. Coutin, N. Victoir, Lévy area for Gaussian processes, preprint, 2005.

[7] A. Dembo, O. Zeitouni, Large Deviations Techniques and Applications, second ed., Applications of Mathematics, vol. 38, Springer-Verlag, New York, ISBN 0-387-98406-2, 1998, xvi+396 pp.

[8] D. Feyel, A. de La Pradelle, Curvilinear integrals along enriched paths, Electronic J. Probab. 11 (2006) 860-892.

[9] P. Friz, Continuity of the Itô-map for Hölder rough path with applications to the Support Theorem in Hölder norm, in: Probability and Partial Differential Equations in Modern Applied Mathematics, in: IMA Volumes in Mathematics and its Applications, vol. 140, 2003 , pp. 117-136.

[10] P. Friz, N. Victoir, Approximations of the Brownian rough path with applications to stochastic analysis, Ann. Inst. H. Poincaré Probab. Statist. 41 (4) (2005) 703-724.

[11] P. Friz, N. Victoir, On the notion of geometric rough paths, Probab. Theory Relat. Fields 136 (3) (2006) 395-416.

[12] P. Friz, N. Victoir, A variation embedding theorem and applications, J. Funct. Anal. 239 (2) (2006) 631-637.

[13] N.C. Jain, G. Kallianpur, A note on uniform convergence of stochastic processes, Ann. Math. Statist. 41 (1970) $1360-1362$.

[14] M. Ledoux, Isoperimetry and Gaussian analysis, in: Lectures on Probability Theory and Statistics, Saint-Flour, 1994, in: Lecture Notes in Math., vol. 1648, Springer, Berlin, 1996, pp. 165-294.

[15] M. Ledoux, Z. Qian, T. Zhang, Large deviations and support theorem for diffusion processes via rough paths, Stochastic Process. Appl. 102 (2) (2002) 265-283.

[16] T.J. Lyons, Differential equations driven by rough signals, Rev. Mat. Iberoamericana 14 (2) (1998) 215-310.

[17] T. Lyons, Z. Qian, System Control and Rough Paths, Oxford University Press, 2002.

[18] A. Millet, M. Sanz-Sole, Large deviations for rough paths of the fractional Brownian motion, Ann. Inst. H. Poincaré Probab. Statist. 42 (2) (2006) 245-271.

[19] A. Millet, M. Sanz-Sole, Approximation of rough paths of fractional Brownian motion, ArXiv-preprint, 2005.

[20] M. Schreiber, Fermeture en probabilité de certains sous-espaces d'un espace $L^{2}$. Application aux chaos de Wiener, Z. Wahrsch. Verw. Gebiete $14(1969 / 70) 36-48$.

\section{Further reading}

[1] L. Decreusefond, A.S. Üstünel, Stochastic analysis of the fractional Brownian motion, Potential Anal. 10 (1997) 177-214. 